Departamento de Educación

Médica, Facultad de Medicina,

Universidad de Concepción.

aBioquímica, Magíster en

Educación Médica para las

Ciencias de la Salud.

bPsicólogo, Magíster en Psicología

con mención en Psicología

Educativa.

'Psicóloga.

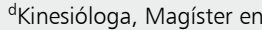

Educación Médica para las Ciencias de la Salud.

eIngeniero Informático, Magíster en Educación Médica para las

Ciencias de la Salud.

fMatrona, Magíster en Educación con mención en Currículum.

Trabajo financiado por el proyecto FONDECYT Regular № 1110718

Recibido el 9 de abril de 2014, aceptado el 29 de septiembre de 2014

Correspondencia a:

Carolina Márquez U.

Departamento de Educación

Médica, Facultad de Medicina,

Universidad de Concepción,

Barrio Universitario $\mathrm{s} / \mathrm{n}$ Concepción, Chile.

Teléfono: 412204932

cmarquezu@udec.cl

\section{Aprendizaje autodirigido y su relación con estilos y estrategias de aprendizaje en estudiantes de medicina}

\author{
CAROLINA MÁRQUEZ U. ${ }^{a}$, EDUARDO FASCE H., \\ CRISTHIAN PÉREZ V. ${ }^{\mathrm{b}}$, JAVIERA ORTEGA B..$^{\mathrm{c}}$, PAULA PARRA P. ${ }^{\mathrm{d}}$, \\ LILIANA ORTIZ M., OLGA MATUS B. ${ }^{e}$, PILAR IBÁÑEZ $G^{f}$
}

\section{Relationship between self-directed learning with learning styles and strategies in medical students}

Background: Self-directed learning (SDL) skills are particularly important in medical education, considering that physicians should be able to regulate their own learning experiences. Aim: To evaluate the relationship between learning styles and strategies and self-directed learning in medical students. Material and Methods: One hundred ninety nine first year medical students (120 males) participated in the study. Preparation for Independent Learning (EPAI) scale was used to assess self-direction. Schmeck learning strategies scale and Honey and Alonso (CHAEA) scales were used to evaluate learning styles and strategies. Results: Theoretical learning style and deep processing learning strategy had positive correlations with self-direct learning. Conclusions: Medical students with theoretical styles and low retention of facts are those with greater ability to selfdirect their learning. Further studies are required to determine the relationship between learning styles and strategies with SDL in medical students. The acquired knowledge will allow the adjustment of teaching strategies to encourage SDL.

(Rev Med Chile 2014; 142: 1422-1430)

Key words: Education, medical; Learning; Models, educational; Students, Medical.
E n el área de la medicina, organizaciones nacionales e internacionales han declarado $\checkmark$ oficialmente que el aprendizaje autodirigido es una variable que debe ser evaluada durante los procesos formativos ${ }^{1-3}$. Las habilidades de aprendizaje autodirigido, vistas como prerrequisitos de capacitación continua, son particularmente importantes en el campo biomédico, donde el conocimiento avanza y cambia continuamente. Se genera así la necesidad que los docentes deban alentar en los futuros médicos la regulación de sus propias experiencias de aprendizaje, ya que esto sería crucial para sus éxitos futuros ${ }^{4-5}$.

El aprendizaje autodirigido toma su base en la andragogía $\mathrm{a}^{6-7}$, sustentada originalmente por Mal- colm Knowles, definiendo a la andragogía como el "arte y ciencia de ayudar a los adultos a aprender", en contraste con la pedagogía concebida como la ciencia de ayudar a aprender a los niños ${ }^{7,8}$.

El aprendizaje autodirigido ha sido referido como un proceso de aprendizaje de carácter estratégico y autoreflexivo, en el cual el alumno toma la iniciativa, con o sin la ayuda de otros, para diagnosticar sus necesidades de aprendizaje, formular sus metas, identificar materiales y recursos humanos para aprender, implementar y elegir las estrategias adecuadas y evaluar los resultados de su propio aprendizaje ${ }^{6,9,10}$. Para implementar esta habilidad se requeriría del uso de estrategias cognitivas, metacognitivas y motivacionales de- 
pendientes de múltiples factores tanto del alumno, como de los docentes, del sistema educativo e incluso de la propia cultura ${ }^{3,6,11}$.

Fisher, King y Tague desarrollaron un instrumento cuyas propiedades psicométricas se han evaluado en estudiantes de distintas carreras del área de la salud, como medicina y enfermería ${ }^{6,12-15}$. Una versión traducida al castellano de esta escala ha mostrado una adecuada validez y confiabilidad al aplicarse en estudiantes de medicina chilenos ${ }^{10}$.

Entre las variables que podrían influenciar esta capacidad se encuentran los 'estilos de aprendizaje', correspondientes a rasgos cognitivos, afectivos y fisiológicos que indican cómo los estudiantes perciben, interaccionan y responden a sus ambientes de aprendizaje ${ }^{16}$, así también como las "estrategias de aprendizaje", dependientes del conjunto de actividades con que el estudiante procesa la información y aborda sus tareas de aprendizaje ${ }^{17}$.

Teóricamente los estilos y estrategias estarían asociados al aprendizaje autodirigido, ya que la capacidad reflexiva-analítica es uno de los componentes necesarios para aprender de manera autónoma, sobre todo entendiendo que los alumnos reflexivos prefieren trabajar solos, pensar detenidamente en el objeto de estudio y establecer conexiones entre sus elementos relevantes ${ }^{18}$. Se suma a lo anterior la capacidad para evaluar las propias necesidades de aprendizaje a fin de definir de manera independiente los objetivos que se perseguirán ${ }^{19}$. Sin embargo, aun existe la incógnita sobre la relación que podría existir entre los estilos y estrategias de aprendizaje con al aprendizaje autodirigido, materia abordada en el presente estudio.

\section{Metodología}

\section{Diseño}

Corresponde a un diseño cuantitativo, no experimental, transversal y correlacional.

\section{Participantes}

Participaron la totalidad de los estudiantes de medicina de la Universidad de Concepción que ingresaron los años $2010(\mathrm{n}=117)$ y 2011 $(\mathrm{n}=118)$. Se excluyó a quienes presentaban más de $5 \%$ de respuestas omitidas y se eliminaron los datos de quienes no entregaron información sobre su sexo $(n=6 ; 3,02 \%)$ y sobre el establecimiento educativo de procedencia $(n=8 ; 4 \%)$. La muestra final quedó constituida por 199 estudiantes de medicina, con 120 hombres $(60,30 \%)$ y 73 mujeres $(36,68 \%)$.

\section{Instrumentos}

Para evaluar la autodirección en los aprendizajes de los alumnos se usó la Escala de Aprendizaje Autodirigido de Fischer, Tague y King (EPAI), traducida al español y validada en alumnos de medicina chilenos por Fasce y cols. ${ }^{10}$. Esta escala está compuesta por 40 ítems que presentan atributos, habilidades o aspectos motivacionales que son característicos de los aprendices autónomos. El participante debe indicar el grado en que el ítem representa una característica de él, utilizando una de cinco alternativas $(1=$ muy en desacuerdo; $2=$ en desacuerdo; 3 = indeciso; $4=$ de acuerdo $y$ $5=$ muy de acuerdo).

Para evaluar estilos de aprendizaje se utilizó el Cuestionario Honey y Alonso (CHAEA) el que cuenta con 80 afirmaciones ante las cuales el sujeto debe responder dicotómicamente, si está de acuerdo (signo "+") o no (signo “-”). Los reactivos se agrupan en cuatro grupos de 20 ítems, cuyas puntuaciones sumativas indican el grado en que el participante presenta uno de los cuatro estilos de aprendizaje propuestos por Honey: activo, reflexivo, teórico y pragmático. Este instrumento ha sido validado previamente y ha sido utilizado en diversos estudios para determinar los estilos de aprendizaje en diversas carreras del área de la salud incluida medicina ${ }^{16,20-23}$.

Para evaluar estrategias de aprendizaje se utilizó la versión adaptada del Inventario de Procesos de Aprendizaje de Schmeck desarrollada por Truffello y Pérez ${ }^{24}$. Este instrumento consta de 55 reactivos en los que se presentan conductas relacionadas con el aprendizaje del alumno, ante las cuales éste debe responder "Verdadero" o "Falso", dependiendo de si la descripción corresponde o no con su propio comportamiento. Los ítems permiten identificar cuatro modelos de aprendizaje: procesamiento elaborativo, estudio metódico, procesamiento profundo y retención de hechos.

\section{Procedimiento}

Para obtener los datos se aplicó a la muestra los tres instrumentos antes mencionados, junto con un cuestionario sociodemográfico. El uso de la información analizada en este estudio fue autorizado por los estudiantes a través un procedimiento de consentimiento informado visado por la Comisión 
Aprendizaje autodirigido, estilos y estrategias de aprendizaje - C. Márquez et al

Nacional de Investigación Científica y Tecnológica de Chile, CONICYT.

\section{Análisis de datos}

Para evaluar la consistencia interna de los instrumentos se usó el coeficiente alfa de Cronbach y para evaluar las relaciones bivariadas el coeficiente de correlación de Pearson. Por último, se utilizó un análisis de regresión lineal múltiple para el análisis multivariado. Se consideró $\mathrm{p}<0,05$ como estadísticamente significativo. En los análisis estadísticos se utilizó el software STATA SE 11.0.

\section{Resultados}

La consistencia interna de los instrumentos presentó niveles adecuados de confiabilidad (Tabla 1).

\section{Análisis bivariados}

Para evaluar la relación entre los estilos de aprendizaje (CHAEA) y las estrategias de aprendizaje (Schmeck) con la escala de aprendizaje autodirigido, se utilizó el coeficiente de correlación de Pearson, en base a un contraste bilateral, comprobándose correlaciones estadísticamente significativas entre algunas puntuaciones (Tablas 2 y 3). Pero estas correlaciones fueron débiles, mostrando un tamaño del efecto pequeño ${ }^{25}$.
En los estilos de aprendizaje, las correlaciones estadísticamente significativas con la escala general y las subescalas del aprendizaje autodirigido fueron (Tabla 2):

- Estilo Teórico con la escala general $(\mathrm{p}<0,05)$, con la subescala Planificación del aprendizaje, $(\mathrm{p}<0,001)$ y con Autoevaluación $(\mathrm{p}<0,05)$.

- Estilo Reflexivo con la subescala Planificación del aprendizaje $(\mathrm{p}<0,05)$ y con el Deseo de aprender $(\mathrm{p}<0,05)$.

- Estilo Pragmático con la subescala Planificación del aprendizaje $(\mathrm{p}<0,05)$ y con Deseo por aprender $(\mathrm{p}<0,05)$.

- Estilo Activo con la subescala de Autoconfianza $(\mathrm{p}<0,01)$.

En el caso de las estrategias de aprendizaje, la Retención de hechos presentó correlaciones inversas y estadísticamente significativas con la escala general de aprendizaje auto dirigido $(\mathrm{p}<0,05)$, y con la subescala de Autogestión $(\mathrm{p}<0,05)$, Tabla 3 .

\section{Análisis multivariado}

Se desarrollaron seis modelos de regresión lineal múltiple en los cuales se evaluó la capacidad predictiva de las estrategias y estilos de aprendizaje considerando cada factor del aprendizaje autodirigido como variable dependiente (Tablas 4 a 8):

Las estrategias y los estilos de aprendizaje

Tabla 1. Estadísticos descriptivos de los instrumentos aplicados a los alumnos de medicina: Aprendizaje Auto dirigido (EPAI), Estrategias de aprendizaje (Schmeck) y Estilos de aprendizaje (CHAEA)

\begin{tabular}{|llccccc|}
\hline \multirow{3}{*}{ EPAI } & Escala & Alfa & M & D.E. & Mín & Máx \\
& Escala general & 0,91 & 154,86 & 14,97 & 69 & 187 \\
& Planificación del aprendizaje & 0,86 & 37,75 & 6,24 & 22 & 50 \\
& Deseo de aprender & 0,81 & 25,92 & 3,31 & 8 & 30 \\
& Autoconfianza & 0,80 & 38,01 & 4,29 & 14 & 45 \\
& Autogestión & 0,71 & 38,21 & 3,75 & 13 & 45 \\
& Autoevaluación & 0,66 & 14,97 & 2,62 & 8 & 20 \\
\multirow{3}{*}{ CHAEA } & Pensamiento elaborativo & 0,63 & 8,13 & 2,17 & 2 & 14 \\
& Estudio metódico & 0,89 & 15,19 & 6,91 & 0 & 27 \\
& Pensamiento profundo & 0,75 & 15,98 & 6,29 & 1 & 28 \\
& Retención de hechos & 0,89 & 13,32 & 7,87 & 1 & 26 \\
& Activo & 0,70 & 11,11 & 3,43 & 2 & 19 \\
& Reflexivo & 0,62 & 15,26 & 2,80 & 3 & 20 \\
& Teórico & 0,65 & 13,24 & 3,02 & 4 & 20 \\
\end{tabular}

$\mathrm{N}=199 ;$ Alfa = coeficiente alfa de Cronbach; $M=$ media; D.E. = desviación estándar; Mín = puntaje mínimo; Máx = puntaje máximo. 
Aprendizaje autodirigido, estilos y estrategias de aprendizaje - C. Márquez et al

Tabla 2. Correlación de los niveles de aprendizaje auto dirigido (EPAI) con los estilos de aprendizaje de los alumnos de medicina $(n=199)$

\begin{tabular}{|lcccc|}
\hline & \multicolumn{2}{c|}{ Estilos de aprendizaje } \\
EPAI & Activo & Reflexivo & Teórico & Pragmático \\
\hline Escala general & 0,06 & 0,13 & $0,22^{* *}$ & 0,13 \\
Planificación del aprendizaje & $-0,11$ & $0,15^{*}$ & $0,29^{* *}$ & $0,15^{*}$ \\
Deseo por aprender & 0,05 & $0,16^{*}$ & 0,10 & $0,16^{*}$ \\
Autoconfianza & $0,21^{* *}$ & 0,02 & 0,11 & 0,02 \\
Autogestión & 0,12 & $-0,01$ & 0,08 & $-0,01$ \\
Autoevaluación & 0,01 & 0,12 & $0,14^{*}$ & 0,12 \\
\hline
\end{tabular}

${ }^{*} p<0,05 ;{ }^{* *} p<0,01 ;{ }^{* *} p<0,001$.

Tabla 3. Correlación de los niveles de aprendizaje autodirigido con las estrategias de aprendizaje de los alumnos de medicina

\begin{tabular}{|lcccc|}
\hline EPAI & $\begin{array}{c}\text { Pensamiento } \\
\text { elaborativo }\end{array}$ & $\begin{array}{c}\text { Estrategias de aprendizaje } \\
\text { Estudio } \\
\text { metódico }\end{array}$ & $\begin{array}{c}\text { Pensamiento } \\
\text { profundo }\end{array}$ & $\begin{array}{c}\text { Retención de } \\
\text { hechos }\end{array}$ \\
Escala general & $-0,05$ & 0,00 & 0,06 & $-0,14^{*}$ \\
\hline Planificación del aprendizaje & $-0,02$ & 0,12 & 0,21 & $-0,10$ \\
\hline Deseo por aprender & $-0,02$ & 0,01 & 0,07 & $-0,04$ \\
Autoconfianza & 0,04 & $-0,07$ & 0,04 & $-0,12$ \\
Autogestión & $-0,06$ & $-0,10$ & 0,02 & $-0,16^{*}$ \\
Autoevaluación & $-0,04$ & 0,01 & $-0,02$ & $-0,09$ \\
\hline
\end{tabular}

$\mathrm{n}=199 ;{ }^{*} \mathrm{p}<0,05 ;{ }^{* *} \mathrm{p}<0,01 ;{ }^{* * *} \mathrm{p}<0,001$

Tabla 4. Resultados de la regresión lineal múltiple para la escala general aprendizaje autodirigido

\begin{tabular}{|lcccc|}
\hline \multicolumn{1}{|c}{} & B & EE & $\boldsymbol{\beta}$ & $\mathbf{s r}^{\mathbf{2}}$ \\
\hline Constante & 123,66 & & & 0,00 \\
\hline Pensamiento elaborativo & $-0,01$ & 0,70 & $-0,00$ & 0,03 \\
\hline Estudio metódico & $0,78^{*}$ & 0,32 & 0,36 & 0,04 \\
\hline Pensamiento profundo & $0,74^{* *}$ & 0,26 & 0,31 & 0,09 \\
Retención de hechos & $-1,28^{* * *}$ & 0,29 & $-0,67$ & 0,01 \\
Estilo activo & 0,64 & 0,36 & 0,15 & 0,00 \\
Estilo reflexivo & 0,30 & 0,40 & 0,06 & 0,02 \\
Estilo teórico & $0,87^{*}$ & 0,43 & 0,18 & 0,00 \\
\hline Estilo pragmático & 0,15 & 0,38 & 0,03 & 0 \\
\hline
\end{tabular}

$\mathrm{R}^{2}=0,17^{* * *} ; \mathrm{R}^{2}$ ajustado $=0,14 \mathrm{~B}=$ coeficientes de regresión no estandarizados; $\mathrm{EE}=$ errores estándar; $\beta=$ coeficientes de regresión estandarizados; $s r^{2}=$ correlaciones semiparciales al cuadrado; $R^{2}=$ coeficiente de determinación; $R^{2}=$ coeficiente de determinación ajustado. $\mathrm{n}=199 ;{ }^{*} \mathrm{p}<0,05 ;{ }^{* *} \mathrm{p}<0,01 ;{ }^{* *} \mathrm{p}<0,001$.

presentaron una relación estadísticamente significativa con el aprendizaje autodirigido en general $(\mathrm{p}<0,001)$, explicando $14 \%$ de su variación $\left(\mathrm{R}^{2}{ }_{\text {adj }}=0,14\right)$. Individualmente, un mayor Estudio metódico $(\mathrm{p}<0,005)$, un mayor Pensamiento profundo ( $\mathrm{p}<0,01)$, y una menor Retención de hechos $(\mathrm{p}<001)$, junto con un estilo de aprendizaje Teórico más acentuado $(\mathrm{p}<0,05)$, se correlacionaron significativamente con el aprendizaje autodirigido, Tabla 4. 
Aprendizaje autodirigido, estilos y estrategias de aprendizaje - C. Márquez et al

Tabla 5. Resultados de la regresión lineal múltiple para la subescala de Planificación del aprendizaje

\begin{tabular}{|lcccc|}
\hline \multicolumn{1}{|c}{} & $\mathbf{B}$ & EE & $\boldsymbol{\beta}$ & $\mathbf{s r}^{\mathbf{2}}$ \\
Constante & 29,61 & & & 0,00 \\
Pensamiento elaborativo & $-0,23$ & 0,28 & $-0,08$ & 0,09 \\
Estudio metódico & $0,61^{* * *}$ & 0,13 & 0,67 & 0,04 \\
Pensamiento profundo & $0,31^{* *}$ & 0,10 & 0,32 & 0,14 \\
Retención de hechos & $-0,68^{* * *}$ & 0,11 & $-0,86$ & 0,00 \\
Estilo activo & 0,04 & 0,14 & 0,02 & 0,00 \\
Estilo reflexivo & 0,01 & 0,16 & 0,00 & 0,03 \\
Estilo teórico & $0,46^{* *}$ & 0,17 & 0,22 & 0,00 \\
\hline Estilo pragmático & $-0,13$ & 0,15 & $-0,07$ & \\
\hline
\end{tabular}

$R^{2}=0,26^{* * *} ; R^{2}$ ajustado $=0,23 \cdot n=199 ;{ }^{*} p<0,05 ;{ }^{* *} p<0,01 ;{ }^{* *} p<0,001$.

Tabla 6. Resultados de la regresión lineal múltiple para la subescala de deseo de aprender

\begin{tabular}{|lcccc|}
\hline \multicolumn{1}{|c}{} & $\mathbf{B}$ & EE & $\boldsymbol{\beta}$ & $\mathbf{s r}^{\mathbf{2}}$ \\
\hline Constante & 19,87 & & & 0,00 \\
\hline Pensamiento elaborativo & $-0,01$ & 0,16 & $-0,00$ & 0,00 \\
\hline Estudio metódico & 0,01 & 0,08 & 0,03 & 0,02 \\
\hline Pensamiento profundo & $0,13^{*}$ & 0,06 & 0,24 & 0,01 \\
\hline Retención de hechos & $-0,09$ & 0,07 & $-0,22$ & 0,01 \\
\hline Estilo Activo & 0,14 & 0,08 & 0,15 & 0,02 \\
Estilo Reflexivo & $0,20^{*}$ & 0,09 & 0,17 & 0,00 \\
\hline Estilo Teórico & 0,09 & 0,10 & 0,08 & 0,00 \\
\hline Estilo Pragmático & $-0,04$ & 0,09 & $-0,04$ & \\
\hline
\end{tabular}

$\mathrm{R}^{2}=0,07 ; \mathrm{R}^{2}$ ajustado $=0,03 . \mathrm{n}=199 ;{ }^{*} \mathrm{p}<0,05 ;{ }^{* *} \mathrm{p}<0,01 ;{ }^{* * *} \mathrm{p}<0,001$.

Tabla 7. Resultados de la regresión lineal múltiple para la subescala de Autoconfianza

\begin{tabular}{|lcccc|}
\hline \multicolumn{1}{|c}{} & B & EE & $\boldsymbol{\beta}$ & $\mathbf{s r}^{\mathbf{2}}$ \\
\hline Constante & 28,61 & & & 0,00 \\
\hline Pensamiento elaborativo & 0,13 & 0,21 & 0,07 & 0,00 \\
Estudio metódico & 0,03 & 0,09 & 0,05 & 0,02 \\
Pensamiento profundo & $0,17^{*}$ & 0,08 & 0,25 & 0,03 \\
Retención de hechos & $-0,21^{*}$ & 0,08 & $-0,38$ & 0,04 \\
Estilo Activo & $0,32^{* * *}$ & 0,10 & 0,25 & 0,00 \\
Estilo Reflexivo & 0,04 & 0,12 & 0,03 & 0,01 \\
Estilo Teórico & 0,20 & 0,13 & 0,14 & 0,00 \\
Estilo Pragmático & 0,09 & 0,11 & 0,06 & \\
\hline
\end{tabular}

$\mathrm{R}^{2}=0,13^{* * *} ; \mathrm{R}^{2}$ ajustado $=0,09 . \mathrm{n}=199 ;{ }^{*} \mathrm{p}<0,05 ;{ }^{* *} \mathrm{p}<0,01 ;{ }^{* * *} \mathrm{p}<0,001$.

Relación entre estrategias y estilos de aprendizaje con las subescalas del aprendizaje auto dirigido:

- Las estrategias y los estilos hicieron una predicción estadísticamente significativa de
$22,96 \%\left(\mathrm{R}_{\text {adi }}^{2}=0,23\right)$ de la Planificación del aprendizaje, $(p<0,001)$. En las estrategias, los predictores estadísticamente significativos fueron un mayor Estudio metódico, $(\mathrm{p}<0,001)$ y Pensamiento profundo, $(\mathrm{p}<0,01)$ y una 
Aprendizaje autodirigido, estilos y estrategias de aprendizaje - C. Márquez et al

Tabla 8. Resultados de la regresión lineal múltiple para la subescala de Autogestión

\begin{tabular}{|c|c|c|c|c|}
\hline & B & EE & $\beta$ & $\mathbf{s r ^ { 2 }}$ \\
\hline Constante & 34,02 & & & \\
\hline Pensamiento elaborativo & 0,13 & 0,19 & 0,08 & 0,00 \\
\hline Estudio metódico & 0,04 & 0,08 & 0,07 & 0,00 \\
\hline Pensamiento profundo & 0,12 & 0,07 & 0,20 & 0,02 \\
\hline Retención de hechos & $-0,20 * *$ & 0,08 & $-0,42$ & 0,03 \\
\hline Estilo Activo & 0,13 & 0,09 & 0,12 & 0,01 \\
\hline Estilo Reflexivo & $-0,03$ & 0,10 & $-0,02$ & 0,00 \\
\hline Estilo Teórico & 0,10 & 0,11 & 0,08 & 0,00 \\
\hline Estilo Pragmático & 0,09 & 0,10 & 0,08 & 0,00 \\
\hline
\end{tabular}

$R^{2}=0,08^{*} ; R^{2}$ ajustado $=0,04 . n=199 ;{ }^{*} p<0,05 ;{ }^{* *} p<0,01 ;{ }^{* *} p<0,001$.

Tabla 9. Resultados de la regresión lineal múltiple para la subescala de Autoevaluación

\begin{tabular}{|lcccc|}
\hline \multicolumn{1}{|c}{} & B & EE & $\boldsymbol{\beta}$ & $\mathbf{s r}^{\mathbf{2}}$ \\
\hline Constante & 11,54 & & & 0,00 \\
\hline Pensamiento elaborativo & $-0,03$ & 0,13 & $-0,02$ & 0,01 \\
\hline Estudio metódico & 0,09 & 0,06 & 0,24 & 0,00 \\
\hline Pensamiento profundo & 0,01 & 0,04 & 0,02 & 0,02 \\
Retención de hechos & $-0,10$ & 0,05 & $-0,29$ & 0,00 \\
Estilo Activo & 0,01 & 0,07 & 0,01 & 0,01 \\
Estilo Reflexivo & 0,07 & 0,07 & 0,08 & 0,00 \\
\hline Estilo Teórico & 0,02 & 0,08 & 0,03 & 0,02 \\
\hline Estilo Pragmático & $0,15^{*}$ & 0,07 & 0,18 & \\
\hline
\end{tabular}

$\mathrm{R}^{2}=0,07 ; \mathrm{R}^{2}$ ajustado $=0,04 . \mathrm{n}=199 ;{ }^{*} \mathrm{p}<0,05 ;{ }^{* *} \mathrm{p}<0,01 ;{ }^{* * *} \mathrm{p}<0,001$.

menor Retención de hechos, $(\mathrm{p}<0,001)$. En los estilos, sólo el Teórico más acentuado fue estadísticamente significativo ( $\mathrm{p}<0,01)$, (Tabla 5).

- Los estilos y las estrategias no hicieron una predicción estadísticamente significativa del nivel de Deseo de aprender, $(\mathrm{p}=0,10)$. A nivel individual fueron predictores estadísticamente significativos un mayor Pensamiento profundo, $(\mathrm{p}<0,05)$, y un estilo Reflexivo más acentuado, $(\mathrm{p}<0,05)$, Tabla 6.

- Las estrategias y los estilos hicieron una predicción estadísticamente significativa de 9,35\% $\left(\mathrm{R}^{2}{ }_{\text {adj }}=0,09\right)$ de la Autoconfianza $(\mathrm{p}<0,001)$. Individualmente, fueron predictores estadísticamente significativos, un mayor Pensamiento profundo, $(\mathrm{p}<0,05)$, una menor Retención de Hechos, $(\mathrm{p}<0,05)$, y un estilo Activo más acentuado, $(\mathrm{p}<0,01)$, (Tabla 7$)$.
- El modelo también resultó estadísticamente significativo para la subescala de Autogestión, ( $\mathrm{p}<0,05)$, explicando $4 \%$ de su variación $\left(\mathrm{R}_{\text {adj }}^{2}=0,04\right)$. Individualmente, sólo una menor Retención de hechos fue un predictor con significación estadística $(\mathrm{p}<0,01)$, (Tabla 8$)$.

- Por último, en el caso de las estrategias, el modelo no logró una predicción estadísticamente significativa para la subescala Autoevaluación, $(\mathrm{p}=0,06)$. Sin embargo, individualmente un estilo Pragmático más acentuado fue un predictor estadísticamente significativo, $(\mathrm{p}<0,05)$, (Tabla 9).

\section{Discusión}

Aun cuando se ha reconocido el efecto favorable que ejerce el aprendizaje autodirigido en los 
Aprendizaje autodirigido, estilos y estrategias de aprendizaje - C. Márquez et al

procesos de enseñanza-aprendizaje ${ }^{9,26}$, en especial por favorecer la autoconfianza, la autonomía, la motivación y la disposición hacia un aprendizaje continuo $^{27}$ y, por tanto, la necesidad de que los docentes adquieran competencias pedagógicas que lo promuevan ${ }^{7,28}$, es escasa la evidencia sobre los factores asociados al aprendizaje autodirigido en la educación médica. Sin embargo, estudios con respecto a la adquisición de idiomas extranjeros en estudiantes de enfermería de Australia, han identificado correlación positiva entre el aprendizaje autodirigido, los estilos de aprendizaje, las estrategias de aprendizaje y el rendimiento académico ${ }^{29}$.

Otros estudios en Estados Unidos de Norteamérica, utilizando el inventario de Kolb, demostraron que enfermeras con estilos convergentes eran aprendices más autodirigidos que los del estilo acomodador, asimilador o divergentes ${ }^{30}$. En otros países como Australia e Irán no se encontraron correlaciones entre los estilos y el SDL ${ }^{27}$.

Los resultados obtenidos en las investigaciones antes señaladas no son susceptibles de comparar con los nuestros debido a que en ellos se utilizaron instrumentos diferentes. Por otra parte, se ha demostrado que los bagajes culturales influencian el aprendizaje autónomo ${ }^{11}$, siendo éste otro factor que dificulta un análisis comparativo.

Nuestros resultados comprueban que los alumnos del estilo Teórico, el cual se ha comprobado predominante en estudiantes de medicina ${ }^{16,20}, y$ que no usan la retención de hechos como estrategia de aprendizaje, son aquellos que se muestran como aprendices más autodirigidos. Ello indica que los alumnos con un estilo de procesamiento centrado en la información conceptual y que a su vez aprenden de manera más sistemática, realizando un análisis más profundo de la información y con menor tendencia al aprendizaje mecánico, son aquellos que reportan una mayor planificación de sus aprendizajes.

Por otra parte, los estudiantes que utilizan estrategias de Estudio metódico y de Pensamiento profundo, son quienes presentan mayores niveles de autonomía, toda vez que presentan una mayor preferencia por el procesamiento conceptual de la información $y$, a su vez, quienes poseen un mayor nivel de sistematicidad en su aprendizaje, pero una menor inclinación por aprender hechos mecánicamente.

La mayor predisposición hacia el procesamiento profundo de la información se vincula a mayores habilidades de planificación de los aprendizajes, mayor deseo por aprender y de autoconfianza.

Siendo la planificación una característica del estilo Teórico, en el cual el desarrollo del pensamiento es secuencial, con alta capacidad de integración de hechos dispares en teorías coherentes, surge como lógica la correlación detectada entre la subescala de planificación con el estilo Teórico y las estrategias de estudio metódico y pensamiento profundo.

La relación entre la subescala de Autoconfianza con el estilo Activo refleja que estos alumnos aprenden de la experiencia y se entusiasman con nuevas cosas, lo que requiere un mayor nivel seguridad en sí mismo y una menor base conceptual. También la subescala de Autoconfianza se relaciona con aprendizajes más profundos y menor retención de hechos, lo que sería esperable dado que estrategias más rudimentarias de aprendizaje están más acordes con sujetos que confían menos en sus propias capacidades.

La subescala de Autoevaluación se correlaciona con el estilo pragmático en donde los estudiantes prefieren buscar ideas y aplicarlas a la realidad, tomando decisiones y explorando una mejor forma de hacer las cosas, acciones que requieren de la capacidad de autoevaluación como medio de verificar cómo realizan sus tareas y el nivel de éxito logrado.

La subescala de Deseo de aprender se asocia a las estrategias de pensamiento profundo y con el estilo reflexivo, demostrando que las estrategias más elaboradas y complejas de aprendizaje, que favorecen el aprendizaje significativo, serían más esperables en estudiantes con un alto nivel de compromiso motivacional con los aprendizajes. Asimismo, estilos más observadores y tranquilos como los reflexivos, exhiben una mayor disposición hacia el proceso de enseñanza aprendizaje. Estos estilos analizan antes de ponerse en marcha al tiempo que se sienten gratificados al aprender nuevos conocimientos.

Los resultados demuestran que es teóricamente coherente que el aprendizaje autodirigido en general, y sus subescalas de Planificación, Autoconfianza y Autogestión, no se relacionen con la retención de hechos, ya que éste es un proceso cognitivo básico y los alumnos con estrategias de estudio autodirigido más desarrolladas utilizan procesos más auto regulados y complejos. 
Aprendizaje autodirigido, estilos y estrategias de aprendizaje - C. Márquez et al

Desde el punto de vista de la gestión educativa, resulta evidente que aquellas metodologías que propicien análisis reflexivo, que generen mayor autoconfianza y determinen una mayor gestión personal, evitando la simple retención de la información, serán aquéllas que en mayor medida contribuyan a desarrollar habilidades de estudio independiente.

Las reflexiones anteriores, deben considerar que aunque significativas las correlaciones no fueron intensas y que la capacidad explicativa del modelo fluctuó entre $4 \%$ y $23 \%$, lo que es bajo pero esperable ante un fenómeno multifactorial como este. Por lo tanto, se requieren nuevos estudios que determinen relaciones entre los estilos y estrategias de aprendizaje en estudiantes de Medicina, además de estudios que consideren otras variables que puedan mediar esta relación. Esto, a fin de adecuar las estrategias de enseñanza a estrategias de aprendizaje que favorezcan el SDL y así promover aprendizajes significativos en nuestros estudiantes. Finalmente, dado que algunas puntuaciones, sobre todo en el CHAEA, resultaron admisibles pero no óptimas $(<0,80)$, se recomienda considerar también mediciones alternativas de la forma en que los estudiantes aprenden.

\section{Referencias}

1. Parra P, Pérez C, Ortiz L y Fasce E. El aprendizaje autodirigido en el contexto de la educación médica. Rev Educ Cienc Salud 2010; 7 (2): 146-51.

2. Pérez C. Diez consejos para promover el aprendizaje autónomo y el compromiso efectivo al enseñar contenidos complejos. Rev Educ Cienc Salud 2010; 7 (1): 50-6.

3. Pérez C, Parra P, Ortiz L, Fasce E. Variables personales y académicas asociadas al aprendizaje autodirigido en la educación médica. Rev Educ Cienc Salud 2010; 7 (2): 152-60.

4. Shokar GS, Shokar MD, Romero CM, Bulik, RJ. Selfdirected Learning: Looking at Outcomes with Medical Students. Family Medicine 2002; 34 (3): 197-200.

5. Gwayali S, Jauhari A, Shankar PR, Saha A, Ahmad M. Readiness for self-directed learning among first semester students or a medical school in Nepal. Journal of Clinical and Diagnostic Research 2001; 5 (1): 20-3.

6. Deyo Z, Huynh D, Rochester C, Sturpe DA, Kiser K. Readiness for Self-directed Learning and Academic Performance in an Abilities Laboratory Course. Am J Pharm Educ 2011; 75 (2) Article 25: 1-6.
7. Hewitt-Taylor J. Self-directed learning: views of teachers and students. Journal of Advanced Nursing 2001; 36 (4): 496-504.

8. Merriam S. Andragogy and Self-Directed Learning: Pillars of Adult Learning Theory. New direction for adult education 2001; N 89.

9. Dynan L, Cate T, Rhee K. The impact of learning structure on students'readiness for self-directed learning. Journal of education for business 2008; 84 (2): 96-100.

10. Fasce E, Pérez C, Ortiz L, Parra P, Matus O. Estructura factorial y confiabilidad de la escala de aprendizaje autodirigido de Fisher, King \& Tague en alumnos de medicina chilenos. Rev Med Chile 2011; 139: 1437-43.

11. Ahmad BE, Majid FA. Self-directed Learning and Culture: A Study on Malay Adult Learners. Procedia Social and Behavioral Sciences 2010; 7 (4): 254-63.

12. Fisher M, King J, Tague G. Development of a selfdirected learning readiness scale for nursing Education. Nurse Education Today 2001; 21: 516-25.

13. Fisher M, King J. The self-directed learning readiness scale for nursing education revisited: A confirmatory factor analysis. Nurse Education Today 2010; 30: 44-8.

14. Hendry G, Ginns P. Readiness for self-directed learning: Validation of a new scale with medical students. Medical Teacher 2009; 31 (10): 918-20.

15. Huynh D, Haines S, Plaza C, Sturpe D, Williams G, Rodríguez M, Roffman D, The Impact of Advanced Pharmacy Practice Experiences on Students' Readiness for Self-directed Learning American Journal of Pharmaceutical Education 2009; 73 (4) Article 65.

16. Borracci RA, Guthman G, Rubio M, Arribalzaga EB. Estilos de aprendizaje en estudiantes universitarios y médicos residentes. Educ Med 2008; 11 (4): 229-38.

17. Fasce E. Aprendizaje profundo y superficial. Rev Educ Cienc Salud 2007; 4 (1): 2.

18. Jennings SF. Personal development plans and selfdirected learning for healthcare professionals: are they evidence based? Postgrad Med J 2007; 83: 518-24.

19. Lindh M. \& Hugo J. Students' reflections on self-directed learning using patient Studies in a master programme in family medicine in South Africa. Education form primary care 2005; 16: 474-81.

20. Palacios S, Matus O, Soto A, Ibáñez P, Fasce E. Estilos de aprendizaje en Primer Año de Medicina según cuestionario Honey-Alonso: publicación preliminar. Rev de Educ Cienc Salud 2006; 3 (2): 22-6.

21. Mc Coll P. Estilos de aprendizaje en los estudiantes de primer año de carreras de la Universidad de Valparaíso, Rev Educ Cienc Salud 2009; 6 (1): 34-41.

22. Nolla M. El proceso cognitivo y el aprendizaje profesional. Educación médica; 2006; 9 (1): 11-6. 
23. Hendry GD, Ginns P. Readiness for self-directed learning: Validation of a new scale with medical students. Medical Teacher 2009; 31: 918-20.

24. Truffello I, Pérez F. Adaptación en Chile del "Inventory of Learning Processes” de Ronald Schmeck. Boletín de Investigación 1988; 6: 109-20.

25. Cohen J. The Analysis of Variance. En: Cohen J. Statistical Power Analysis for The Behavioral Sciences. Hillsdale: Erlbaum 1988; p. 273-406.

25. Cunningham J. Self-Direction: A Critical Tool in Distance Learning. Common Ground Journal 2010; 7: 89-100.

26. El-Gilany AH, Abusaad FES. Self-direct learning readiness and learning styles among Saudi undergraduate nursing students. Nurse Education Today 2013; 33 (9) 1040-4.

27. Loyens S, Magda J, Rokers R. Self-Directed Learning in Problem-Based Learning and its Relationships with SelfRegulated Learning. Educ Psychol Rev 2008; 20: 411-27.

28. D’Amore A, James S, Mitchell E. Learning styles of firstyear undergraduate nursing and midwifery students: A cross-sectional survey utilising the Kolb Learning Style Inventory. Nurse Education Today 2012; 32: 506-15.

29. Cid S. El uso de estrategias de aprendizaje y su correlación con la motivación de logro en los estudiantes. Revista Iberoamericana sobre Calidad, Eficacia y Cambio en Educación 2008; 6: 101-20. 